\title{
Die Form- und metrischen Verhältnisse der sog. 4 wirbeligen Kreuzbeine bei den Japanern.
}

\author{
Von \\ Kotaro Fujino. \\ Aus dem Anatomischen Institut der Medizinischen Akademie zu Kioto.
}

Mit 3 Textfiguren.

Mit dem Japanerkreuzbeine haben sich vom anthropologischen Standpunkte aus, und zwar besonders bezüglich der metrischen Verhältnisse, schon viele Autoren beschäftigt, so Hasebe ${ }^{1)}$, Miyamoto2), Okamoto ${ }^{3)}$ und $\mathrm{Arai}^{4}$. Was die numerischen Variationen des Kreuzbeins anbetrifft, so herrscht bei den Japanern das 5 wirbelige vor. Das 6 wirbelige ist viel weniger häufig ${ }^{5)}$ und das 4 wirbelige selten. Das 4 wirbelige Sakrum findet sich von vielen Autoren als selten angegeben: Nach Paterson ${ }^{6}$ $-2.6 \%$, nach Radlauer ${ }^{7)}-2.8 \%$; Adolphi ${ }^{\text {s) }}$ fand unter 234 Sakra ein,

1) Hasebe, K., Die Wirbelsäule der Japaner. Zeitschrift f. Morphologie u. Anthropologie, Bd. 15, 1912.

2) II f amoto, H., Anthropological Studies on the Skeleton of the Japanese. The Journal of the Anthropological Society of Tokyo, Vol 42, 1927 (japanisch).

3) Okamoto, T., Anthropological Studies on the Skeleton of the Japanese (japanisch) The Journal of the Anthropological society of Tokyo, Vol. 45, Supplement 2, 1930.

4) Arai, M., On the Pelvis of the Japanese (japanisch). The Journal of the Anthropological society of Tokso, Vol. 4S, 193:.

5) Buto, T., Die Formverhältnisse der 6 wirbeligen Kreubeine bei den Japanern. Folia anatomica japonica, 13d. 10. 1932.

6) Paterson, zit. nach Radlauer.

7) Radlauer, C., Beiträge zur Anthropologie des Kreuzbeines. Morpholog. Jahrbuch, Bd. 38. 1908.

8) Adolphi, H., Über den Bau des menschlichen Kreuzbeines u. die Verschiedenheit seiner Zusammensetzung in Prag u. Jurjew-Dorpat. Morpholog. Jahrbuch, Bd. 44, 1912. 
Fischel ${ }^{1)}$ unter 5243 und Fret $z^{2)}$ unter 1133104 wirbelige Krenzbeine. Bei den Japanerkreuzbeinen fand Hasebe unter 181 Wirbelsäulen 2 Fälle von 4 wirbeligem Kreuzbeine, also 1.1\%, Okamoto unter 50 Kreuzbeinen ein vierwirbeliges, also $2 \%$. Bei der Durchmusterung der in hiesigen Institut aufbewahrten 172 Wirbelsäulen mit exakten Wirbelformeln fand ich keinen einzigen Fall von ausgesprochenem 4 wirbeligem Kreuzbeine (im Sinne von Fretz's Gruppe 1). Dafür stiess ich auf 3 Fälle, die man eventuell als 4 wirbelige Sakra (im Simne von Fretz's Gruppe 5 und 6) auffassen könnte. Es handelt sich um die folgenden zwei Kategorien :

a) 4 Sakralwirbel und ein Lumbosakralübergangswirbel 2 Fälle,

b) 4 Sakralwirbel und ein sakrokokzygealer Übergangswirbel 1 Fall

Diese Kategorie a resp. b entsprechen der Gruppe 5 resp. 6 von Fretz. Was die Häufigkeit der hierhergehörigen Fälle anbelangt, so findet sich in Fretz's Tabelle 1 folgendes:

\begin{tabular}{c|c|c} 
& $\begin{array}{c}\text { Unter } \\
1732 \text { Sakra }\end{array}$ & $\begin{array}{c}\text { unter } \\
740 \text { Sakra }\end{array}$ \\
\hline $\mathrm{a}=$ Gruppe 5 & 2 & 2 \\
$\mathrm{~b}=$ Gruppe 6 & - & 5
\end{tabular}

Danach sind die von mir gefundenen Fälle nicht allzu häufig. Im folgenden habe ich die drei Kreuzbeine formanalytisch und metrisch studiert.

\section{Formverhältnisse.}

Fall 1. Ein mit einem Lumbosakralübergangswirbel von überwiegendem Lumbalcharakter versehenes 4 wirbeliges Kreuzbein.-56 j. \&, Nr. 122 (Fig. 1).

Das Sakrum stammt von einer Wirbelsäule mit folgender Formel :

$$
(1-7) \mathrm{C}(8-19) \mathrm{T}(20-23) \mathrm{L} \text { (24)LS + (25-28)S }(29-32) \mathrm{Co} .
$$

Der 24. Wirbel stellt einen Lumbosakralübergangswirbel dar. Die Fornverhältuisse dieses Wirbels hat bereits Toyoda ${ }^{3)}$ in seiner diesbezüg-

1) Fischel, A., Untersuchungen über die Wirbelsäule und den Brustkorb des Menschen. Anatomische Hefte, Bd. 31, Heft 95, 1906.

2) Fret\%, G. P., Das menschliche Sakrum. Morpholog. Jahrkuch, Bd. 48, 1914.

3) Toyoda, J., Über den lumbosakralen Ủbergangswirbel bei den Japanern. Folia anatomica Japonica, Bd. 5, Heft 1/2, 1927. 
lichen Abhandlung beschrieben. Das Sakrum (Fig. 1) besitzt in seiner ventralen und seinen beiden seitlichen Ansichten (b, c) Lumbalcharakter, und zwar sowohl hinsichtlich der Formbeschaffenheit des Körpers, des Dorsalbogens und der Gelenkfortsätze als auch bezüglich der Verbindung mit dem entsprechenden Anteile des nächstfolgenden Wirbels. Das einzige sakrale Zeichen des Wirbels findet sich erst an dem Seitenfortsatz. Der Seitenfortsatz weist ausser seinem eigentlichen proximalwärts und zugleich etwas nach hinten und neben dem oberen Gelenkfortsatze sich erhebenden Vorsprung noch einen distal- und zugleich ventralwärts sich

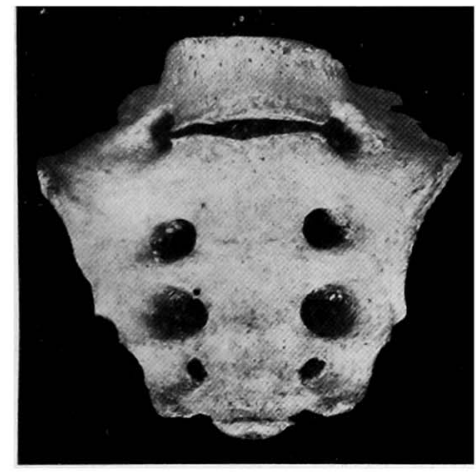

a

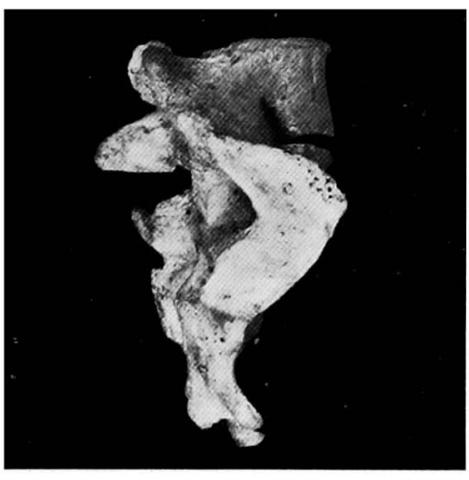

b

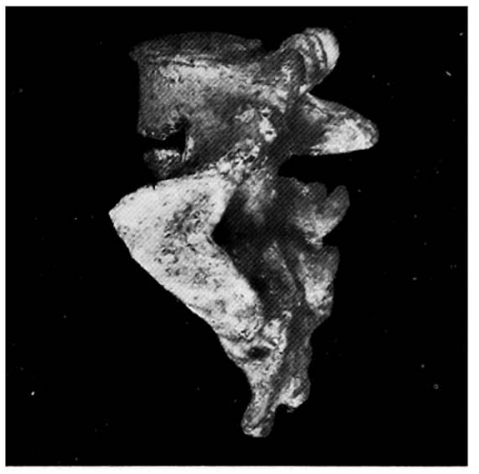

(:

Fig. 1. (56 jähr. ㅇ )

a ventrale-, b rechtsseitige-, c linksseitige Ansicht.

erstreckenden auf. IVie Rosenber( ${ }^{1)}$ bereits ausgesprochen, handelt es sich bei diesem letztgenannten Torsprung um ein bei der Sakralisation besonders entstandenes Umbildungsgebilde. Der Torsprung (Fig. 1 b, c) erstreckt sich gegen die Ala sacri, die dem nächstfolgenden Wirbel (d. h. 25.) ihr Dasein verdankt, koalesziert mit dieser, beteiligt sich jedoch nicht an der Bildung der Facies auricularis. Die Facies auricularis wird durch den 25. und 26. Wirbel gebildet, während sich der 27. Wirbel nur andeutungsweise an dieser Bildung beteiligt.

Auf Grund der obengenannten Formbeschaffenheiten kamn man die Sakralisierungsumbildung so auffassen, dass die Sakralisationsvorgänge von der gewöhnlichen oberen Schwelle (25. Wirbel) aus ein wenig proximalwärts fortgeschritten sind, d. h. der 24. Wirbel gerade jetzt dazu neigt, sich an der Sakralisation zu beteiligen. Das Sakrum lässt ein doppeltes Promontorium erkennen. Der Canalis sacralis ist vorn 2. bis

1) Rosenherg, E., Die vergleichende Form der Wirbelsäule des Menschen und ihre Bedeutung. 1. Teil. 1920. Jena. 
zum letzten Wirbel geschlossen. Der proximale Rand der Pars lateralis sacri zeigt Hypobasalität. Das distale Ende der Pars lateralis sacri, wo der laterale und der distale Sakrumrand zusammentreffen, spitzt sich ziemlich weit distalwärts $\% u$. Das deutet bekanntlich darauf hin, dass sich erst ganz kürzlich der nächstfolgende Wirbel (d. h. der 29. Wirbel bezw. 1. Kaudalwirbel) vom Sakrum abgetrennt hat. So sind auch hier, an dem distalen Sakrumende die Sakralisationsvorgänge von der gewöhnlichen unteren Schwelle (29. Wirbel) einen Schritt weiter proximalwärts fortgeschritten, d. h. die distale Scluwelle selbst (29. Wirbel) hat sich als der 1. Kaudalwirbel vom Sakrum abgetrennt.

Danach handelt es sich bei dem betreffenden Sakrum nicht um ein wesentlich 5 wirbeliges, sondern um ein wesentlich 4 wirbeliges, dessen proximales Ende mil einem überwiegend Iumbalcharakter aufweisenden Wirbel versehen ist.

Fall 2. Ein mit einem Lumbosakralübergangswirbel versehenes 4 wirbeliges Sakrum von asymmetrischer Form-Erwachsener Mann, Nr. 55. (Fig. 2).

Das Sakrum stammt aus einer Wirbelsäule von folgender Wirbelformel :

$$
(1-7) \mathrm{C}(8-19) \mathrm{T}(20) \mathrm{TL} .(21-24) \mathrm{L}(25) \mathrm{LS}+(26-29) \mathrm{S}(30-33) \mathrm{Co} .
$$

Bei dem 25. Wirbel handelt es sich um einen Lumbosakralübergangswirbel von asymmetrischer Form, der bezüglich seiner Formbeschaffenheit ron Toyoda in einer speziellen Abhandlung näher beschrieben worden ist. Dieser Wirbel (Fig. 2) besitzt bei der ventralen (a) und den beiden seitlichen (b, c) Ansichten in seinen verschiedenen

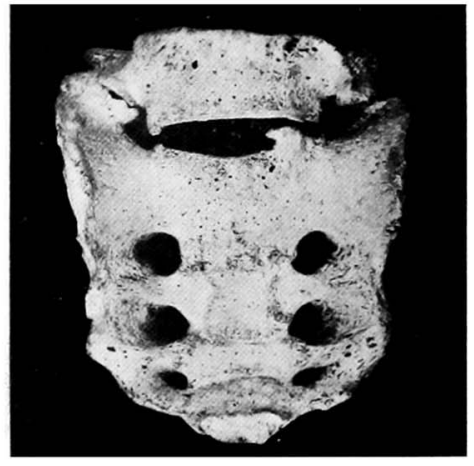

a

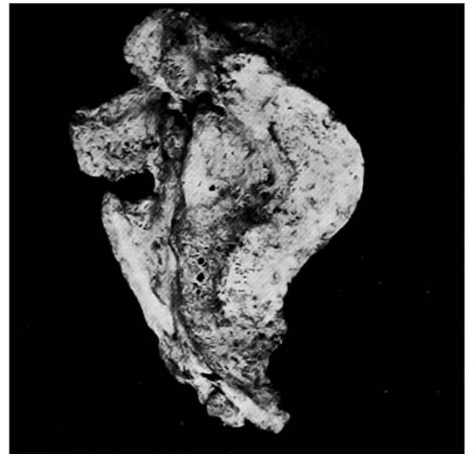

$\mathrm{b}$

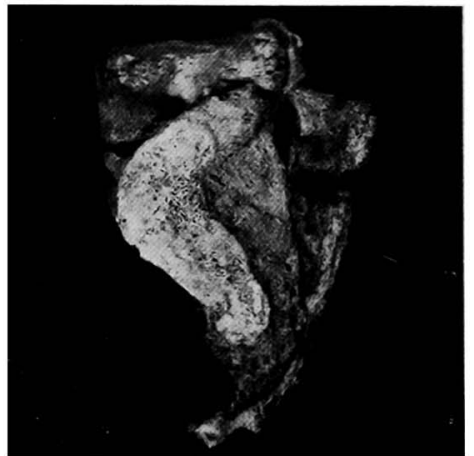

c

Fig. 2. (Erwachsener Mann)

a ventrale, b rechtsseitige, c linksseitige Ansicht. 
Abschnitten bis auf den rechtsseitigen Seitenfortsatz Lumbalcharakter. Der rechtsseitige Seitenfortsatz lässt sich als ein Umbildungszeichen bei der Sakralisation auffassen, weil sein distal und zugleich mehr ventralwärts sich erstreckender Vorsprung mit dem proximalen Abschnitt der Ala sacri, die ihr Dasein hauptsächlich dem Seitenteil des 26. Wirbels verdankt, synostotisch verbunden ist. Die gewöhnliche obere Schwelle der Sakralisation ist beim vorliegenden Falle um einen Schritt distalwärts zurückgerückt, indem die Schwelle (d. h. der 25. Wirbel) gewissermassen ihren Sakralcharakter verloren hat und dafür Lumbalcharakter angenommen hat und es sogar aussieht, als wolle sich der 25. Wirbel von der oberen Sakrumschwelle aus proximalwärts abtrennen. Das Sakrum weist ein doppeltes Promontorium auf, und der Sakralkanal ist vom 26. Wirbel an bis zum Gebiet des letzten (29.) Wirbels geschlossen. Der proximale Rand der Pars lateralis sacri besitzt Hypobasalität. Am distalen Rand der Pars lateralis sacri deutet nichts auf distalwärts fortschreitende Sakralisation hin. Nach all dem Obigen handelt es sich bei diesem Sakrum nicht um ein 5 wirbeliges im strengen Sime, sondern ist jede Hälfte besonders beschaffen. Auf der rechten Seite, wo der Seitenfortsatz des 25. Wirbels ziemlich deutlichen Sakralcharakter aufweist und an der Bildung der Facies auricularis teilnimmt, imponiert das Sakrum als 5 wirbeliges (b), auf der linken Seite dagegen, wo der Seitenfortsatz ausgesp:ochenen Lumbalcharakter besitzt, als 4 wirbeliges (c). So ist also, formanalytisch betrachtet, das betreffende Sakrum nur auf der rechten Hälfte als ein wesentlich 5 wirbeliges aufzufassen, d. h. auf dieser Seite umfasst die Sakralisierumbildung 5 Wirbel. Auf der linken Seite dagegen ist die obere Schwelle des Spielraumes für die Sakralisierumbildung im Vergleich mit der rechten eine Wirbelstrecke weit distalwärts zurückgeblieben, und zwar beschränkt sich hier die Sakralisierumbildung nur auf 4 Wirbel (26.-29.). Deswegen kam man das Sakrum hier auf der linken Seite als ein 4 wirbeliges ansehen, oder mit anderen Worten, der 25. Wirbel auf der linken Seite ist als letzter Isumbalwirbel rom Sakrum losgetrennt worden.

Fall 3. Ein mit einem Sakrokokzygealübergangswirbel versehenes 4 wirbeliges Sakrum von asymmetrischer Form. 54 jähr. $\hat{o}$. Nr. 10. (Fig. 3).

Das Sakrum stammt aus einer Wirbelsäule von folgender Formel :

$$
(1-7) \mathrm{C}(8-19) \mathrm{T}(20-25) \mathrm{L}(26-29) \mathrm{S}+(30) \mathrm{SCo}(31-33) \mathrm{Co} .
$$

Das Sakrum besitzt anstatt 87 Foramina sacralia. So ist das distalste Foramen auf der rechten Seite von lateralher unterbrochen (Fig. 3, a, 
b). Der letzte Sakralwirbel, d. h. der 30. Wirbel, ist ein Sakrokokzygealübergangswirbel. Über die Formverhältnisse des betreffenden Wirbels hat bereits Toyoda $\mathrm{a}^{1)}$ in seiner diesbezüglichen Abhandlung geschrieben. Das Sakrum besitzt ein einfaches Promontorium und die Pars lateralis Hyperbasalität. Formanalytisch betrachtet, ist das Sakrum asymmetrisch, und zwar hinsichtlich des letzten Wirbels. Der 30. Wirbel besitzt in

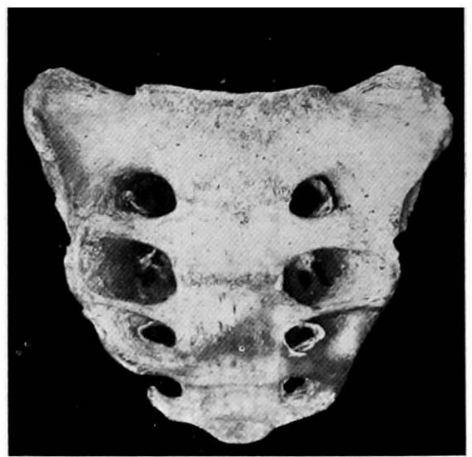

a

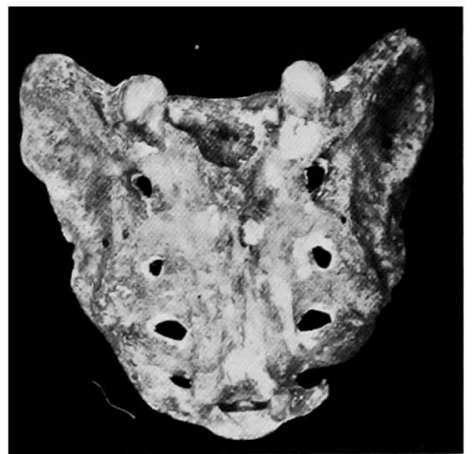

b)

Fig. 8. ( $5 \pm$ jähr. ô $)$

a ventrale, b dorsale Ansicht.

ler rechtsseitigen Ansicht ausgesprochenen Kokzygealcharakter, und zwar sowohl in seiner Pars lateralis als auch im Cornu coccygis. Sogar der Wirbelkörper ist mit dem des vorangehenden Wirbel nicht ganz synostotisch rerbunden. Auf der linken Seite imponiert dagegen der Wirbel als Sakralwirbel. Nach dem Obigen kam man dieses Sakrum hinsichtlich des Spielraumes der Sakralisicrumbildungsvorgänge wie folgt auffassen. Vor allem ist hier die obere Schwelle des Spielraumes der Umbildungsvorgänge im Vergleich mit lem growönlichen Fall auf beiden Seiten eine Wirbelstrecke weit distalwärts zurückgeblieben, so dass anstatt des 25. Wirbels (gewöhnlicher Fall) erst der 26. Wirbel sakralen Charakter aufweist. Die untere Schwelle des Umbildungsbereiches ist jedoch auf beiden Seiten verschieden. Auf der linken Seite ist die untere Schwelle wie dic obere eine Wirbelstrecke weit kaudalwärts zurückgeblieben (bis zum 30. Wirbel). So imponiert dieses Sakrum hinsichtlich der Sakralisationsvorgänge als 5 wirbeliges, und zwar als eines, das eine Wirbelstrecke kaudalwärts zurückgeblieben ist. Anf ler rechten Seite befindet sich die untere Schwelle an ihrer gewöhnlichen Stelle (29. Wirbel), so

1) Toyoda, J., Über den sakrokok\%ygealen Übergangswirbel bei den Japanern. Folia anatomica Japonica, Bis. 4. 1926. 
dass das Sakrum hier als 4 wirbeliges aufzufassen ist. Der 30. Wirbel ist hier als 1. Kokzygealwirbel gelegentlich mit dem Sakrum verwachsen.

\section{Metrische Verhältnisse.}

Bezüglich der metrischen Verhältnisse der abnormen Formen des Sakrums, und zwar des mit dem Lumbosakralübergangswirbel versehenen, erwähnte Wetzel, ${ }^{1)}$ dass alle Sakra mit Lumbosakralübergangswirbel kürzer als die normalen seien. Ich habe in der folgenden Tabelle 1 zunächst die von mir an den auffallenden Sakren gefundenen Masse zusammengestellt. Weiter habe ich auf Grund dieser Resultate eine Abweichungsfigur (Tabelle 2) nach Martin hergestellt, wobei ich als Grundwerte Okamoto's Messresultate, die dieser Forscher an KinaiJapaner-Sakra gefunden hat, benutzte.

1) Wotzel, G., Studien an australischen Kreuzbeinen. Arehiv f. Anthropologie, $\mathrm{N}$ F, $1:$, 1914 . 
Tabbelle 1.

\begin{tabular}{|c|c|c|c|}
\hline & Fall 1 & Fall 2 & Fall 3 \\
\hline Bogenlänge & $87 \mathrm{~mm}$ & $85 \mathrm{~mm}$ & $100 \mathrm{~mm}$ \\
\hline Vordere gerade Jänge & 80 & 72 & 85 \\
\hline Obere Bogenbreite & 112 & 94 & 113 \\
\hline Obere gerade Breite & 109 & 87 & 104 \\
\hline Grösste Bogenhöhe & 13 & 21 & 23 \\
\hline $\begin{array}{l}\text { Fntfernung der Jage der grössten } \\
\text { Bogenhöhe vom Promontrium }\end{array}$ & 59 & 49 & 56 \\
\hline Mittlere Bogenbreite & 90 & 90 & 86 \\
\hline Mittlere gerade Breite & 89 & 87 & $8 \ddot{2}$ \\
\hline Untere gerade Breite & 49 & 59 & 53 \\
\hline $\begin{array}{l}\text { Median sagittaler Durchmesser der } \\
\text { Basis des Kreu\%beins }\end{array}$ & 29 & 35 & 31 \\
\hline $\begin{array}{l}\text { (irösster transversaler Durchmesser } \\
\text { der Basis des Kreuzbeins }\end{array}$ & 43 & 46 & 49 \\
\hline Promontoriumwinkel & 73 & 63 & 55 \\
\hline $\begin{array}{l}\text { Längo der Facies auricularis: Rechts } \\
\text { links }\end{array}$ & $\begin{array}{l}46 \\
52\end{array}$ & $\begin{array}{l}66 \\
68\end{array}$ & $\begin{array}{l}51 \\
52\end{array}$ \\
\hline $\begin{array}{l}\text { Breite der Facies auricularis: Rechts } \\
\text { links }\end{array}$ & $\begin{array}{l}29 \\
31\end{array}$ & $\begin{array}{l}24 \\
25\end{array}$ & $\begin{array}{l}27 \\
27\end{array}$ \\
\hline $\begin{array}{l}\text { Tiefe d. oheren öffnumg des Canalis } \\
\text { sacralis }\end{array}$ & 12 & 15 & 16 \\
\hline $\begin{array}{l}\text { Breite d. oberen iffnung des Can- } \\
\text { alis sacralis }\end{array}$ & 24 & 25 & 26 \\
\hline Iängenbreiten-Index & 136.25 & 120.83 & 122.35 \\
\hline Bogenlängen-gerade Breite-Index & 125.29 & 102.35 & 104. \\
\hline Sehnenhöhen-Index & 16.25 & 29.16 & 27.06 \\
\hline $\begin{array}{l}\text { IIöhenlage-Index der Krewzbein- } \\
\text { krïmmung }\end{array}$ & 73.75 & 68.06 & 65.88 \\
\hline Bogen-Sehnen-Index & 91.95 & 84.71 & 85. \\
\hline Oberer (Querkrümmungs-Index & 97.32 & 92.55 & 92.04 \\
\hline
\end{tabular}


4 wirbelige Kreuzbeine bei den Japanern.

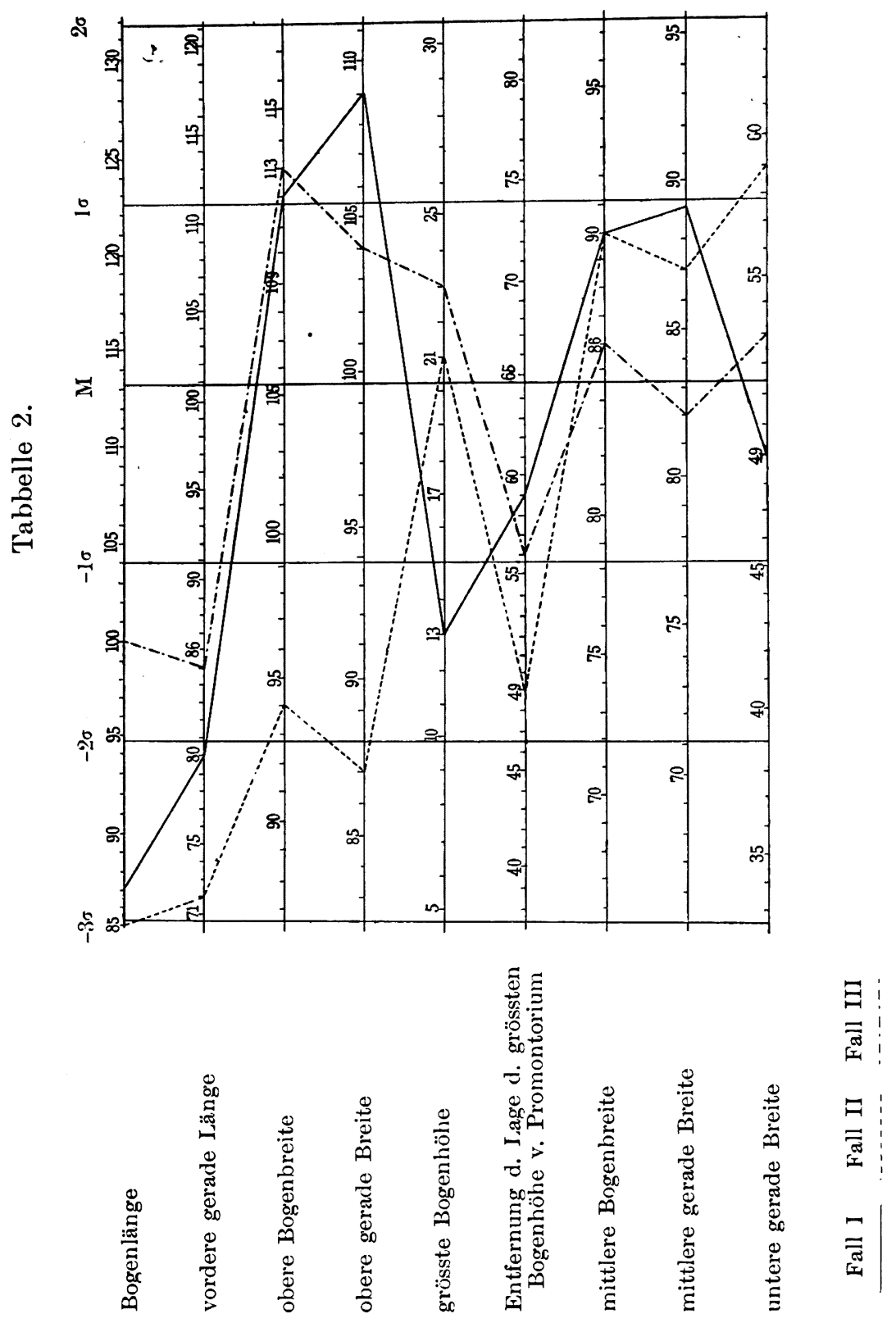

